\section{Editorial}

Check for updates

\section{OPEN ACCESS}

Received: Dec 9, 2019

Accepted: Dec 23, 2019

Address for Correspondence:

Youngkeun Ahn, MD, PhD, FACC, FSCAl

Department of Cardiology, Cardiovascular

Center, Chonnam National University Hospital

671 Jebong-ro, Dong-gu, Gwangju 61469,

Republic of Korea.

E-mail: cecilyk@hanmail.net

(c) 2020 The Korean Academy of Medical

Sciences.

This is an Open Access article distributed under the terms of the Creative Commons Attribution Non-Commercial License (https:// creativecommons.org/licenses/by-nc/4.0/) which permits unrestricted non-commercial use, distribution, and reproduction in any medium, provided the original work is properly cited.

ORCID iDs

Min Chul Kim (D)

https://orcid.org/0000-0001-6026-1702

Youngkeun Ahn (D)

https://orcid.org/0000-0003-2022-9366

Funding

This article was supported by a grant of the Bio \& Medical Technology Development Program of the NRF funded by the Korean government, MSIP (2017M3A9E8023020).

Disclosure

The authors have no potential conflicts of interest to disclose.

\title{
The Value of Exercise Stress Test in Patients with Stable Ischemic Heart Disease
}

\section{Min Chul Kim (1) and Youngkeun Ahn (1)}

Division of Cardiology, Department of Internal Medicine, Chonnam National University Medical School, Gwangju, Korea

- See the article "Long-Term Outcomes in Patients Undergoing Percutaneous Coronary Intervention with or without Preprocedural Exercise Stress Test” in volume 35, number 1, e3.

Percutaneous coronary intervention (PCI) is the standard treatment for patients with severe coronary artery stenosis. The current guidelines state that all patients with acute coronary syndrome should receive invasive coronary angiography, and those with severe coronary artery stenosis should then undergo PCI or bypass surgery. On the contrary, PCI did not improve either the prognosis or functional capacity to an extent greater than that afforded by optimal medical therapy (OMT) for patients with stable ischemic heart disease (SIHD) in either the COURAGE or ORBITA trials.1,2 However, patients in the COURAGE trial exhibited only low-level ischemia, and PCI employed bare-metal stents, which are currently rarely used. The ORBITA trial assessed functional capacity for only 6 weeks. Therefore, any benefit of ischemia-driven PCI for SIHD patients for whom non-invasive stress tests are positive remains unclear.

In the current issue, Kim et al. $^{3}$ compared hard clinical endpoints (all-cause death or myocardial infarction [MI]) between a test group for whom an exercise stress test (EST) was positive within 180 days prior to PCI, and a non-stress-tested group; the study ran for 5.6 years. Patients who underwent elective PCI after a positive EST were at a reduced risk of all-cause death and MI compared to those who underwent PCI without the EST. However, the rates of all revascularization procedures were similar in both groups. Although patients for whom the EST was positive exhibited the best prognosis, those for whom the EST was negative evidenced a better prognosis than the untested group. Almost all patients (94\%) were at moderate-to-high risk as indicated by the EST.

Thus, the EST has been a useful diagnostic tool in patients with suspected SIHD, and the previous guidelines recommended that, when possible, the EST should be used for risk stratification of all SIHD patients. Although the value of EST was decreased to rule-in or rule-out significant coronary artery disease in the recent guideline, an EST remains useful in selected patients, complementing clinical evaluation of ischemic symptoms, exercise tolerance, arrhythmia induction, the blood pressure response, and risk assessment. ${ }^{4}$

The results of the International Study of Comparative Health Effectiveness With Medical and Invasive Approaches (ISCHEMIA) trial were recently published. ${ }^{5}$ This trial is the largest to date comparing invasive and conservative strategies for SIHD patients who evidenced ischemia on non-invasive stress tests; initial invasive treatment did not reduce the risk of hard 
clinical endpoints to a greater extent than an initial conservative strategy. Of all patients, $25 \%$ took the EST; $87 \%$ evidenced moderate-to-high ischemia. On subgroup analysis, the outcomes did not differ significantly by the extent of baseline ischemia. The trial thus reconfirmed that routine PCI did not reduce the risk of adverse clinical outcomes in SIHD patients, in line with the findings of previous randomized trials.

The current study was retrospective in nature; the groups differed in terms of baseline characteristics; and many unmeasured confounding factors were in play. Critically, the reason why the EST was not scheduled for some patients remains unclear, as the authors mention in the limitations section. Given the results of the ISCHEMIA trial and earlier trials, the choice of PCI or OMT for SIHD patients should be dictated by ischemic symptoms, not the extent of myocardial ischemia evident on a non-invasive EST.

Despite these limitations, the study reveals the long-term benefits of the EST in SIHD patients who underwent elective PCI. The principal objective of this study was to explore the long-term clinical outcomes of such patients undergoing elective PCI with or without a prior EST, not to compare PCI and OMT prescribed for those with myocardial ischemia. Although the EST is less useful for SIHD patients then others, selected patients benefited from such testing. EST can be easily performed in the outpatient department; this may not be the case for non-invasive stress tests involving imaging. Further study is needed to determine the optimal candidates for EST among SIHD patients.

\section{REFERENCES}

1. Boden WE, O'Rourke RA, Teo KK, Hartigan PM, Maron DJ, Kostuk WJ, et al- Optimal medical therapy with or without PCI for stable coronary artery disease. N Engl J Med 2007;356(15):1503-16. PUBMED | CROSSREF

2. Al-Lamee R, Thompson D, Dehbi HM, Sen S, Tang K, Davies J, et al- Percutaneous coronary intervention in stable angina (ORBITA): a double-blind, randomised controlled trial. Lancet 2018;391(10115):31-40. PUBMED | CROSSREF

3. Kim J, Lee JM, Park TK, Yang JH, Song YB, Choi JH, et al. Long-term outcomes in patients undergoing percutaneous coronary intervention with or without preprocedural exercise stress test. J Korean Med Sci 2020;35(1):e3. PUBMED | CROSSREF

4. Knuuti J, Wijns W, Saraste A, Capodanno D, Barbato E, Funck-Brentano C, et al· 2019 ESC Guidelines for the diagnosis and management of chronic coronary syndromes. Eur Heart J2019. DOI: 10.1093/eurheartj/ ehz425.

PUBMED | CROSSREF

5. American Heart Association. ISCHEMIA (International Study of Comparative Health Effectiveness With Medical and Invasive Approaches) Clinical Trial Details. http://professional.heart.org/professional/ ScienceNews/UCM_505226_ISCHEMIA-Clinical-Trial-Detatils.jsp. Updated 2019. Accessed December 8, 2019. 